\title{
Outcomes of lensectomy and risk factors for failure in spherophakic eyes with secondary glaucoma
}

\author{
Divya P Rao, ${ }^{1}$ Praveen J John, ${ }^{1}$ Mohammed Hasnat Ali, ${ }^{2}$ Ramesh Kekunnaya, ${ }^{3}$ \\ Subhadra Jalali, ${ }^{4}$ Chandra Sekhar Garudadri, ${ }^{1}$ Sirisha Senthil ${ }^{1}$
}

'VST Centre for Glaucoma, LV Prasad Eye Institute, Hyderabad, India

${ }^{2}$ Department of Clinical Epidemiology and Biostatistics, LV Prasad Eye Institute, Hyderabad, India

${ }^{3}$ Jasti V Ramanamma Children's Eye Care Centre, L V Prasad Eye Institute, Hyderabad, India

${ }^{4}$ Srimathi Kanuri Santhamma Vitreoretinal Services, L V Prasad Eye Institute, Hyderabad, India

\section{Correspondence to}

Dr Sirisha Senthil, L V Prasad

Eye Institute, Kallam Anji Reddy campus, Road No: 2, Banjara Hills, Hyderabad 500034, India; sirishasenthil@|vpei.org

Received 12 June 2017 Revised 9 August 2017 Accepted 16 August 2017

Published Online First

19 September 2017
Check for updates

To cite: Rao DP, John $\mathrm{PJ}$ Ali $\mathrm{MH}$, et al. Br J Ophthalmol 2018:102:790-795.

\section{ABSTRACT}

Purpose To report the outcomes of lensectomy in spherophakic eyes with subluxated or dislocated crystalline lenses and secondary glaucoma.

Methods Lensectomy was performed in 52 eyes, 36 eyes with lens subluxation and 16 eyes with lens dislocation with secondary glaucoma from 1991 to 2016. Glaucoma was diagnosed based on intraocular pressure $(I O P) \geq 22 \mathrm{~mm} \mathrm{Hg}$ and/or glaucomatous optic disc damage. Complete success was defined as IOP $\geq 5$ and $\leq 21 \mathrm{~mm} \mathrm{Hg}$ without antiglaucoma medications (AGMs) or surgery, and eyes needing oral AGM or surgical intervention for IOP control or those with complication causing loss of light perception were considered failure. Results Median (IQR) age at lensectomy was 12 (6-18) years, and median spherical equivalent was $-14.5 \mathrm{D}$ (-23.7to -13). Median follow-up was 30.6 (5.4-103.4) months. Median logMAR (logarithm of minimal angle of resolution) visual acuity improved from 0.95 (0.6-1.8) to $0.4(0.2-1.3)$ after lensectomy $(p=0.01)$. Median IOP decreased from $22 \mathrm{~mm} \mathrm{Hg}(17-31)$ to $14 \mathrm{~mm} \mathrm{Hg}(11-19)$ at final follow-up $(p=0.01)$. Median number of AGM decreased from $2(2-3)$ to $1(0-2)$ at final follow-up $(p<0.0001)$, and glaucoma surgery was needed in four eyes (7.7\% eyes). Complete success probability was $69 \%$ at 1 year and $51 \%$ at 5 years. Younger age ( $<6$ years), higher presenting IOP (>32 mm Hg) and larger cup to disc ratio at presentation were found to be significant risk factors for failure.

Conclusion Lensectomy was effective in controlling IOP in close to half of all eyes with spherophakia and secondary glaucoma, $40 \%$ eyes needed AGM and only $7.7 \%$ eyes needed glaucoma surgery for IOP control. In this cohort, younger age, higher IOP and larger cup to disc ratio at presentation were risk factors for poor glaucoma control after lensectomy.

\section{INTRODUCTION}

Spherophakia is a rare, often bilateral condition, in which the crystalline lens assumes a spherical shape due to abnormally lax zonules. Spherophakic lenses have reduced equatorial diameter and increased anteroposterior diameter causing high lenticular myopia. ${ }^{12}$ The increased anteroposterior diameter of the lens and its anterior displacement due to weak zonules predisposes these eyes to acute pupillary block or chronic angle closure glaucoma. The triad of angle closure glaucoma, shallow anterior chamber and refractive myopia in a young patient are described as hallmarks of this condition. ${ }^{34}$ The most important cause of permanent visual loss in spherophakia is glaucoma, ${ }^{2}$ angle closure being the most common cause. ${ }^{3-7}$ Weak zonules in these eyes often lead to lens subluxation or dislocation resulting in complications such as elevated intraocular pressure (IOP), corneal decompensation and rarely retinal detachment. ${ }^{2}$ Although subluxation or dislocation of the lens causing acute or chronic angle closure is the primary cause of elevated IOP, open angles with developmental angle anomaly also contribute to decrease in aqueous outflow and elevated IOP. ${ }^{9}$ Management options for subluxated or dislocated lenses with elevated IOP include lens removal alone or combining lens extraction with glaucoma surgery. ${ }^{7-17}$ Controversy exists on the role of lensectomy alone in lowering IOP in spherophakic eyes. Some studies have reported no benefit, and few case reports have shown IOP control in the early postoperative period, however needing additional medications or surgery in the long term. Muralidhar et al and Yasar reported no benefit on IOP reduction following lensectomy ${ }^{10}{ }^{17}$; on the contrary, lens extraction with or without intraocular lens implantation was found to be effective in decreasing IOP in few studies with short follow-up. 79 14-16 However, Yang et al have reported long-term IOP elevation needing additional antiglaucoma medications (AGMs) or glaucoma surgery to control IOP. ${ }^{14}$ There are no studies that have looked at the long-term outcomes and risk factors for predicting long-term failure in eyes with subluxated or dislocated lenses in eyes with spherophakia and secondary glaucoma.

\section{METHODS}

We retrospectively reviewed records of consecutive patients who underwent lensectomy for subluxated or dislocated lens in eyes with spherophakia and secondary glaucoma over a 25 -year period (19912016). The study was approved by the Institutional review board of L V Prasad Eye Institute and adhered to the tenets of Declaration of Helsinki. Informed consent was taken from the parents of all subjects prior to the intervention. All patients diagnosed with spherophakia and secondary glaucoma who underwent pars plana lensectomy or lensectomy through a limbal route were included. Patients with prior filtering surgery or those who underwent combined glaucoma filtration surgery with lensectomy or scleral fixated intraocular lens (SFIOL) or glued IOL or lens extraction with in-the-bag IOL placement were also excluded. The eyes that were medically treated or under observation without lensectomy were not included in our study. 


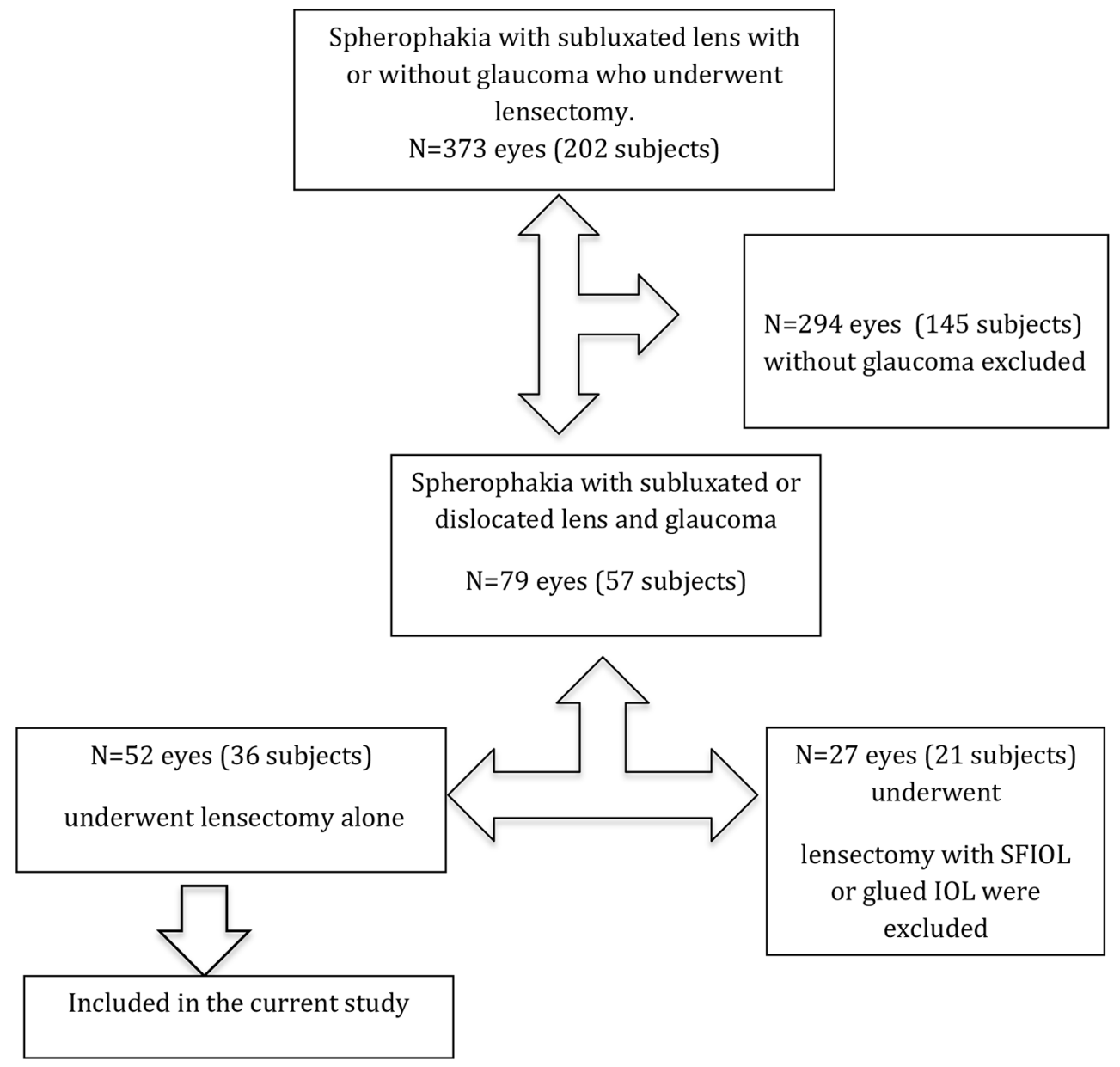

Figure 1 Flow chart showing the patient selection, inclusion and exclusion criteria

The variables collected included age, gender, presenting complaint, associated systemic conditions, preoperative and postoperative best-corrected visual acuity (BCVA), preoperative and postoperative refraction, IOP at presentation (IOP was measured either with Goldmann applanation tonometer or Perkins tonometer), preoperative and postoperative IOP, number of preoperative and postoperative AGMs used, laser peripheral iridotomy, lens status (subluxation/dislocation) at presentation, gonioscopy, cup to disc ratio, indication for lensectomy, technique of lensectomy, complications of the procedure or any other surgical intervention and duration of follow-up.

Spherophakia was diagnosed clinically based on the spherical shape of the lens on slit lamp and the visibility of the lens equator on pharmacological mydriasis (or with indirect ophthalmoscopy when posteriorly dislocated), with high refractive myopia (when possible). For the purpose of the study, glaucoma was defined based on one or more of the following criteria: (1) IOP $\geq 22 \mathrm{~mm}$ $\mathrm{Hg}$ or IOP $\leq 22 \mathrm{~mm} \mathrm{Hg}$ on AGM and (2) glaucomatous optic disc damage. Elevated IOP $\geq 22 \mathrm{~mm} \mathrm{Hg}$ in the absence of disc damage is ocular hypertension; however, in eyes with secondary glaucoma, we often refer to this as glaucoma, hence we have combined ocular hypertension and glaucoma and would refer to them as glaucoma in the manuscript.

\section{Surgical technique}

Lensectomy was performed by pars plana approach or limbal route. In the pars plana approach, two/three sclerotomies were performed, $3 \mathrm{~mm}$ from limbus (or age appropriate distance) in the inferotemporal, superotemporal and superonasal quadrants. Infusion cannula was fixed in the inferotemporal quadrant. Complete lensectomy was performed using a vitreous cutter making sure the capsular remnants were removed. Limited anterior vitrectomy was also performed up to anterior one-third of vitreous cavity. At the end of the surgery, peripheral retinal examination was performed to rule out any inadvertent retinal breaks. The sclerotomies were closed with 8-0 vicryl suture. In recent times, the pars plana vitrectomy (PPV) and lensectomy are performed with 23/25 gauge surgery after technological advancements. In the limbal approach, two paracenteses were performed at 2'0 clock or 10'0 clock position for the vitrectomy probe and at $6^{\prime} 0$ clock position for the anterior chamber maintainer. A complete lensectomy was performed with limited anterior vitrectomy. All surgical sites were closed with 10-0 nylon sutures. The sutures were removed after 1-6 weeks in most cases. The limbal route or pars plana route for lensectomy was based on the position of the subluxated or dislocated lens, the status of the cornea and the surgeon's preference.

Complete success was defined as IOP $\leq 21$ and $\geq 5 \mathrm{~mm} \mathrm{Hg}$ without the need for AGM. Qualified success was defined as IOP $\leq 21$ and $\geq 5 \mathrm{~mm} \mathrm{Hg}$ with topical AGMs. The eyes with medically uncontrolled IOP $\geq 22 \mathrm{~mm} \mathrm{Hg}$ requiring oral AGM to achieve IOP control or those requiring additional glaucoma procedures to lower IOP $<21 \mathrm{~mm} \mathrm{Hg}$ or a complication resulting in loss of light perception were labelled as failure. 
Table 1 Demographic, preoperative and postoperative clinical details of eyes with spherophakia and glaucoma who underwent lensectomy ( $n=52$ eyes of 36 subjects)

\begin{tabular}{llll}
\hline Parameters & $\begin{array}{l}\text { Preoperative median } \\
(\text { IQR })\end{array}$ & $\begin{array}{l}\text { Postoperative } \\
\text { median (IQR) }\end{array}$ & p Value \\
\hline Age (years) & $12(6-18)$ & & \\
Gender (M: $F)$ & $21: 15$ & $10.6(9.4-13)$ & \\
SE in dioptre $(n=32)$ & $-14.5(-23.75$ to -13$)$ & $0.4(0.2-1.3)$ & $<0.001$ \\
\hline VA by logMAR $(n=42)$ & $0.95(0.6-1.8)$ & $14(11-19)$ & $<0.001$ \\
\hline IOP (mm Hg) $(n=50)$ & $26(20-37)$ & $1(0-2)$ & $<0.001$ \\
$\begin{array}{l}\text { Median AGM }(n=48) \\
\text { Cup to disc ratio }(n=44)\end{array}$ & $2(2-3)$ & & \\
$\begin{array}{l}\text { Median follow- } \\
\text { up (months) }\end{array}$ & $30.6(5.35-0.8)$ & & \\
\hline
\end{tabular}

AGM, antiglaucoma medication; F, female; IOP, intraocular pressure; log MAR, logarithm of minimal angle of resolution; $\mathrm{M}$, male; $\mathrm{SE}$, spherical equivalent; $\mathrm{VA}$, visual acuity.

\section{Statistical analysis}

Descriptive statistics included mean and SD for normally distributed variables and median and IQR for non-normally distributed variables. Kaplan-Meier survival curves were used to assess cumulative probability of success. A multivariate Cox regression model with stepwise elimination using Akaike information criteria (AIC) was used to assess the risk factors for failure. A $\mathrm{p} \leq 0.05$ was considered statistically significant. Statistical analysis was performed using commercial software (Stata V.12.0; StataCorp and R software).

\section{RESULTS}

Between 1991 and 2016, 373 eyes of 202 subjects with spherophakia underwent lensectomy at our institute. During this period, 79 eyes out of 57 subjects had glaucoma in spherophakic eyes with subluxated or dislocated lens. Of these, 52 eyes of 36 subjects underwent lensectomy, which were included in our study. Lensectomy with SFIOL or glued IOL was performed in 27 eyes of 21 subjects, and these were excluded from the analysis.

\section{Demographic, preoperative and postoperative parameters}

Demographic, preoperative and postoperative parameters are described in table 1 . There were 21 male $(58.3 \%)$ and 15 female (41.6\%) subjects. Systemic associations were noted in 13 subjects, 11 had Weill Marchesani syndrome and 1 each had Marfan's syndrome and homocystenemia. The most common presenting complaint was decrease in vision in 23 subjects, 6 subjects presented with painful diminution of vision, 2 subjects had photophobia, 3 came for discomfort in one eye and 1 had family history of glaucoma and came for routine eye examination.

Preoperative refractive error data $(n=32)$ revealed a median spherical equivalent of -14.5 dioptre (D) (IQR: -13 to -23.75 ; range: $-4.5 \mathrm{D}$ to $-39.25 \mathrm{D})$. The median log MAR (logarithm of minimal angle of resolution) BCVA at presentation was 0.95 (IQR: 0.6-1.8). The median IOP at presentation $(\mathrm{n}=50)$ was $26 \mathrm{~mm} \mathrm{Hg}$ (IQR: 20-37; range: $10-63 \mathrm{~mm} \mathrm{Hg}$ ); in two eyes with severe corneal oedema with anterior lens subluxation, digital IOP was recorded high. Preoperatively, 48 out of the 52 eyes were on topical AGM, 5 eyes were on 4 AGMs, 13 eyes were on 3 AGMs, 23 eyes were on 2 AGMs and 7 eyes were on 1 AGM. Median number of preoperative AGM was 2 (2-3). Median preoperative IOP ( $n=48$ eyes) before undergoing lensectomy was $22 \mathrm{~mm} \mathrm{Hg}$ (IQR: 17-31). Gonioscopy showed closed angles in 19 eyes and open angles in 10 eyes, and in 23 eyes, gonioscopy was not possible or was not available. Eight eyes had undergone prior laser peripheral iridotomy. Crystalline lens was subluxated in 37 eyes, anteriorly dislocated in 12 eyes and posteriorly dislocated in 3 eyes. The median cup to disc ratio $(n=44$ eyes) was 0.6 (IQR: $0.35-0.8$ ). Glaucomatous disc damage was present in 23 eyes, 21 eyes had normal disc with IOP $>21 \mathrm{~mm} \mathrm{Hg}$ and the remaining 8 eyes had elevated IOP, but disc details were not available.

\section{Lensectomy}

The median age (IQR) at lensectomy was 12 years (IQR: 6-18 years). Bilateral lensectomy was performed in 16 subjects. Five eyes underwent lensectomy by limbal route, and 47 eyes underwent lensectomy by pars plana route.

Preoperative and postoperative parameters are shown in table 1. Median follow-up after lensectomy was 30.6 months (5.5-103). Median postoperative logMAR visual acuity at last follow-up ( $\mathrm{n}=49$ eyes) improved from $0.95(0.6-1.8)$ at presentation to 0.4 (IQR: 0.2-1.3) $(\mathrm{p}=0.001)$. Postoperatively, median spherical equivalent $(n=48)$ was 10.6 D (IQR: 9.4-13 D). The median preoperative IOP was $26(20-37) \mathrm{mm} \mathrm{Hg}$. This reduced significantly at final follow-up IOP to $14 \mathrm{~mm} \mathrm{Hg}$ (IQR: 11-19; $\mathrm{p}=0.001)$. Median number of AGM at last follow-up was 1 (0-2), which was significantly less compared with the preoperative AGMs of $2(2-3)(\mathrm{p}<0.001)$. Following lensectomy, 23 eyes (44.2\%) had IOP control without any AGM, additional 21 eyes requiring one or more AGMs (40.4\%) for IOP control. It is interesting to note that 14 out of the $21(66.6 \%)$ eyes that needed AGM did so within 3 months after lensectomy. Failure was seen in eight eyes $(15.4 \%$; 95\% CI 6.9 to 28.1$)$ due to uncontrolled IOP in seven eyes and posterior segment complication with decrease in vision in one eye. Of the 8 eyes that failed, 4 eyes underwent glaucoma surgery for IOP control, 3 eyes were controlled with oral AGMs and 1 eye developed retinal detachment (RD) with no perception of light with nil visual improvement despite intervention.

The cumulative complete success probability of lensectomy in eyes with spherophakia and glaucoma by Kaplan-Meier analysis was $71 \%$ at 3 months, (number at risk 'n' $=32$ ) $69 \%$ at 1 year $(\mathrm{n}=28)$ and $50.9 \%$ at 5 years $(\mathrm{n}=18)$ (figure 2$)$. The cumulative qualified success probability was $100 \%$ at 1 year $(n=34)$, which was maintained until 3 years $(n=28)$ and was $88 \%$ at 5 years $(n=25)$ (figure 3).

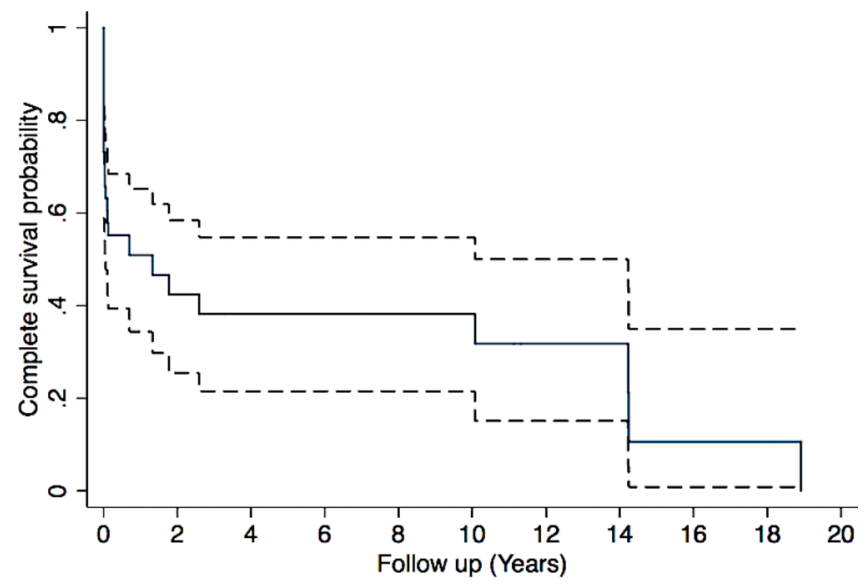

Figure 2 Kaplan-Meier curve showing the complete success probability (solid line with $95 \%$ Cls as shown by dotted lines) of lensectomy in eyes with glaucoma in spherophakia. 


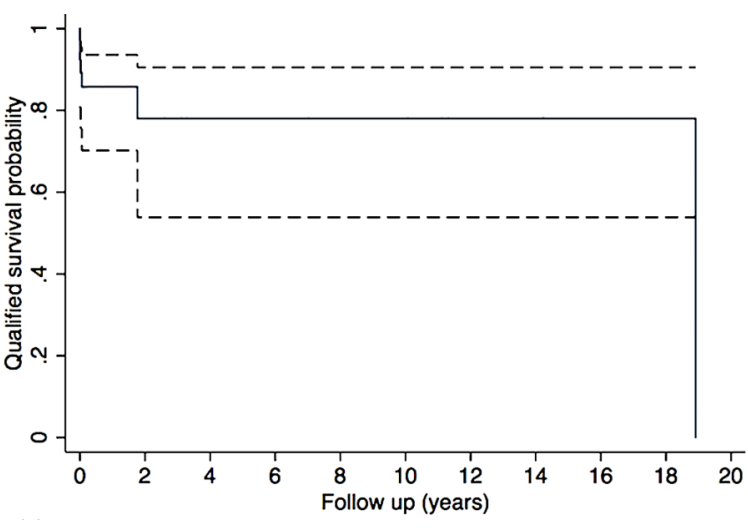

Figure 3 Kaplan-Meier curve showing the qualified success probability (solid line with $95 \% \mathrm{Cls}$ as shown by dotted lines) of lensectomy in eyes with glaucoma in spherophakia.

It is interesting to note that among the 15 eyes, which had dislocated lenses either anterior or posterior, 12 had complete success, 2 had qualified success and only 1 eye had failure.

Postlensectomy complications included choroidal detachment (CD) in three eyes and all responded to intensive topical steroids and topical cycloplegics, and RD in one eye. The eye with RD underwent belt buckle with pars plana vitrectomy and silicone oil injection. The final visual acuity of this eye was light perception despite attached retina.

Risk factors for predicting need for AGM or glaucoma surgery A multivariate Cox regression model with stepwise elimination using AIC was used to assess the effect of preoperative characteristics on the risk of failure of lensectomy for glaucoma control. The factors considered in the model were age, sex, BCVA at presentation, preoperative IOP, cup to disc ratio at presentation, subluxation or dislocation of lens, preoperative number of AGM, angle status and presence of peripheral iridotomy.

The final model retained age, preoperative IOP, cup to disc ratio and number of AGM as the risk factors (table 2). Age was divided into three clusters by quantile distribution. Cluster A ranged from 2 to 6 years, cluster $\mathrm{B}$ ranged from 7 to 18 years and cluster $\mathrm{C}$ was $\geq 19$ years at the time of surgery. Younger age had high risk for postoperative elevated IOP compared with age older than 6 years (older age was protective, $7-18$ years $1 / 0.13=7.6$ times and $>19$ years $=1 / 0.12=8.33$ times). By K-means clustering technique, the preoperative IOP was divided into three groups: group A: IOP less than $19 \mathrm{~mm} \mathrm{Hg}$, group B: IOP 20-32 $\mathrm{mm} \mathrm{Hg}$ and group C: IOP $>32 \mathrm{~mm} \mathrm{Hg}$. IOP $>32 \mathrm{~mm}$ $\mathrm{Hg}$ at presentation was a significant risk factor with $\mathrm{HR}$ of 6.27 in univariate model and 4.1 in multivariate model. Higher cup to disc ratio at presentation was also a significant risk factor with 22.2 times greater risk for failure on multivariate model. Higher number of preoperative AGM (>2 AGMs) was a risk factor for failure in univariate model but not in multivariate model.

Angle status and laser PI were not associated with failure. Of the 19 eyes with closed angles on gonioscopy, in 12 eyes, IOP was controlled after lensectomy, 4 eyes needed AGM for IOP control and 3 eyes needed glaucoma surgery for IOP control. Of the 10 eyes with open angles, 4 eyes had good IOP control after lensectomy, 5 eyes needed AGM and 1 eye needed trabeculectomy for IOP control.

\section{DISCUSSION}

Glaucoma is the most common blinding complication in spherophakia. In the largest retrospective study by Senthil et al, glaucoma was diagnosed in $51 \%$ of the eyes at presentation, majority were young and a large proportion of them were blind due to glaucomatous optic neuropathy. ${ }^{7}$ This emphasises the need to detect and treat glaucoma early and appropriately to prevent visual impairment and blindness associated with this condition.

Lens position abnormality plays a significant role in causing glaucoma in eyes with spherophakia. Hence, removing the lens should play an important role in decreasing IOP; however, there are conflicting reports in the literature. The current study, to our knowledge, is the largest series with 52 eyes and 30.6month median follow-up, reporting outcomes of lensectomy in spherophakic eyes with subluxated or dislocated lenses associated with secondary glaucoma. Our long-term follow-up results and the risk factor analysis, we believe, would add new knowledge to the existing literature.

In our study, following lensectomy in eyes with spherophakia and secondary glaucoma, there was significant improvement

Table 2 Risk factors for failure (increase in IOP or need for glaucoma surgery)

Cox's proportional hazard model on time to event (risk factors for complete failure)

\begin{tabular}{|c|c|c|c|c|}
\hline & \multicolumn{2}{|l|}{ Univariate } & \multicolumn{2}{|l|}{ Multivariate } \\
\hline & Crude HR $(95 \% \mathrm{Cl})$ & Crude $p$ value & $\begin{array}{l}\text { Adjusted HR } \\
(95 \% \mathrm{Cl})\end{array}$ & $\mathrm{p}$ Value \\
\hline \multicolumn{5}{|l|}{$\begin{array}{l}\text { Age }^{*} \text { : } \\
\text { Reference=cluster A }\end{array}$} \\
\hline Cluster B & $0.49(0.2$ to 1.17$)$ & 0.109 & $0.13(0.03$ to 0.5$)$ & 0.003 \\
\hline Cluster C & 0.63 (0.21 to 1.92$)$ & 0.419 & 0.12 (0.03 to 0.55$)$ & 0.006 \\
\hline \multicolumn{5}{|l|}{$\begin{array}{l}\text { Preoperative IOPt: } \\
\text { reference=group A }\end{array}$} \\
\hline Group B & 1.04 (0.3 to 3.57$)$ & 0.947 & 0.38 (0.1 to 1.5$)$ & 0.169 \\
\hline Group C & 6.27 (1.92 to 20.43$)$ & 0.002 & 4.1 (1.08 to 15.54$)$ & 0.038 \\
\hline CDR (preoperative) & 1.4 (0.24 to 8.08$)$ & 0.708 & 22.21 (1.68 to 93.$)$ & 0.019 \\
\hline No of AGM (preoperative) & 1.72 (1.09 to 2.72$)$ & 0.02 & 1.62 (0.93 to 2.8 ) & 0.086 \\
\hline
\end{tabular}

*Preoperative age was divided into three clusters by quantile distribution. Cluster A ranged from 2 to 6 years, cluster B ranged from 7 to 18 years and cluster $\mathrm{C}$ was $\geq 19$ years age during surgery.

†By K-means clustering technique, the preoperative IOP was divided into three groups. Group A: IOP less than $19 \mathrm{~mm} \mathrm{Hg}$, group B: IOP 20-32 mm Hg and group C: IOP >32 mm $\mathrm{Hg}$.

AGM, antiglaucoma medication; CDR, cup to disc ratio; IOP, intraocular pressure. 
in visual acuity, IOP control was noted in half of all eyes over 5 years without need for AGM. The number of AGM significantly decreased postlensectomy and close to $40 \%$ eyes had IOP control with AGM, only $7.7 \%$ eyes needed glaucoma surgery for IOP control.

Glaucoma in spherophakia can be multifactorial. ${ }^{7}$ Elevated IOP could be due to acute or chronic angle closure due to lens position abnormality or may have open angles with angle anomaly or dysgenesis. ${ }^{7}$ When glaucoma is associated with lens position abnormality such as subluxated or dislocated lenses, various surgical modalities have been recommended based on isolated case reports, either by lensectomy, extracapsular cataract extraction (ECCE), phacoemulsification, SFIOL, trabeculectomy, shunt surgeries or a combination of these. ${ }^{9-20}$

There are limited and conflicting reports on the outcome of lensectomy alone in the treatment of glaucoma associated with spherophakia. Willoughby and Wishart reported IOP control with only lens extraction (phacoemulsification) in two eyes of a patient with chronic pupillary block and patent PI with 4 to 6 months follow-up. ${ }^{9}$ Kaushik et al reported a case of bilateral acute angle closure glaucoma where following lensectomy, IOP was under control without AGM until 6 weeks. ${ }^{19}$ Another report by Tailor showed good IOP control over 2 years in two spherophakic eyes after ECCE and intraocular lens implantation. ${ }^{20}$

In a study by Dagi and Walton, eight spherophakic eyes with subluxated lenses, elevated IOP and angle closure, lensectomy was useful to control IOP and prevent progression to complete angle closure in eyes that were operated early. Two of the cases in their series who underwent delayed lensectomy had persistent elevated IOP even after lensectomy. Hence, they concluded that early lensectomy was beneficial for IOP control in spherophakic eyes with subluxated lenses by preventing synechial angle closure. $^{21}$

In an earlier study by Senthil et al, out of the 14 eyes with elevated IOP that underwent lensectomy, 50\% eyes had IOP control, the rest needed AGM to control IOP and none needed glaucoma surgery to control IOP. ${ }^{7}$ Another study by the same group showed that, following trabeculectomy in spherophakic eyes with glaucoma, $45 \%$ of the eyes required lensectomy in the postoperative period. ${ }^{18}$ It was noted that majority of these eyes that needed lensectomy had subtle subluxation of the lens prior to trabeculectomy. Hence, they concluded that in the presence of subluxated lens and high IOP, lensectomy would be a better choice rather than a trabeculectomy.

Few studies have reported lensectomy alone to be ineffective in controlling IOP in eyes with spherophakia and elevated IOP. In a study by Muralidhar et al, out of the 16 eyes in which lensectomy was performed for dislocated lens, four eyes with preoperative elevated IOP continued to have high IOP postlensectomy, and all four eyes needed surgical intervention for IOP control. Hence, they concluded that lensectomy was not beneficial in controlling IOP in spherophakic eyes. ${ }^{10}$ They also reported that glaucoma surgery was also not successful in controlling IOP in these eyes, and many needed cyclodiode as a second intervention. Possible delayed presentation was responsible for refractory nature of glaucoma in their study, although they have not explained the reason for the refractory nature of the eyes in their cohort.

Transient complications were seen in three eyes (choroidal detachment), which improved with conservative management. Hypotony postsurgery could have led to the complication. One eye had retinal detachment, a sight threatening complication. Retinal detachment following pars plana lensectomy and vitrectomy is an uncommon but known complication due to the retinal breaks that develop intraoperatively or in the late postoperative period, ${ }^{22}$ in predisposed patients with Marfan's syndrome. ${ }^{23}$ Our patient had no syndromic association, had subluxated lens and had an uneventful surgery. Although the complication was detected and surgically managed, the visual acuity did not improve.

We found younger age at presentation, higher IOP, more number of AGM (in univariate model) use and greater disc damage at presentation to be significantly associated with higher risk of failure, that is, need for AGM or surgical intervention for IOP control after lensectomy. Closed angles on gonioscopy and need for laser PI could be a parameter that could be associated with failure of IOP control after lensectomy. However, there was no association between laser PI or angle status with development of glaucoma in our study. This observation is similar to what was noted in a study by Muralidhar et $a l^{10}$ In their study, following lensectomy, 2/6 eyes that required trabeculectomy had open angles, trabeculectomy failed and both eyes needed retrabeculectomy and ultimately needed trans-scleral cyclophotocoagulation for IOP control. This further justifies that observation that although angle closure is the most common presentation, eyes with open angles with possible developmental angle anomaly could be associated with refractory glaucoma in eyes with microspherophakia. In our series, although lensectomy helped decrease IOP in 4/10 eyes with open angles, the rest needed additional medications or surgery for IOP control strengthening the hypothesis of angle dysgenesis in these eyes. It is possible that the subluxated or dislocated lens were also partly contributing to the rise in IOP which could explain IOP control with lensectomy in some of these eyes.

One of the limitations of our study is its retrospective design with its inherent problems and variable follow-up. However, for a rare condition such as spherophakia, to evaluate the longterm outcomes and risk factors in a uniform cohort of eyes with secondary glaucoma and subluxated or dislocated lenses, a retrospective study is valuable. Another limitation is incomplete data on gonioscopy for close to $40 \%$ of the eyes. Among the $56 \%$ eyes where gonioscopy was available, majority of the eyes had closed angles and in the risk factor analysis, the angle status did not turn out to be a risk factor, this could possibly be related to developmental angle anomaly in eyes with open angles that contributed to glaucoma and also due to less sample size. The extent of angle closure was not documented which may have helped us to look for association between extent of angle closure and failure. Not including eyes that underwent any other lens procedure such as SFIOL/glued IOL or combined surgeries could be a limitation. However, for the uniformity of the cohort and to decrease the variations in the outcomes that would be related to different surgical procedures, we excluded them.

If lensectomy alone can control IOP in spherophakic eyes with glaucoma and lens position abnormality, trabeculectomy which is associated with serious complications such as shallow/flat AC and its sequelae can be avoided. In our series, after lensectomy, half of all the eyes had well-controlled IOP, additional $40 \%$ eyes had IOP control with AGM. Sight threatening complication (RD) was noted in only one eye. Based on these results, in spherophakic eyes with subluxated or dislocated lenses and elevated IOP, we recommend lensectomy as the procedure of choice. AGM or glaucoma intervention could be planned if and when needed. Younger children with IOP $>32 \mathrm{~mm} \mathrm{Hg}$ with higher cup to disc ratio and need for higher number of AGM preoperatively predicted need for AGM or glaucoma surgery after lensectomy. Subjects with these risk factors should be counselled about need for additional AGM or glaucoma surgery after 
lensectomy. Close follow-up in the postoperative period is necessary to monitor IOP since majority of those that needed AGM required them within 3 months after lensectomy. The role of early lensectomy in prophylaxis against angle closure glaucoma in these eyes would also need to be evaluated.

Correction notice This article has been corrected since it was published Online First. The spelling of author name "Ramesh Kekunaya" has been corrected to "Ramesh Kekunnaya".

Contributors DPR and PJJ contributed in the data collection, interpretation of data and writing of the manuscript. MHA helped with data analysis and interpretation of data. RK, SJ and CSG contributed in critically revising the manuscript for important intellectual content and final approval of the version to be published. SS contributed in the conception, design, acquisition, analysis, interpretation of data and drafting of the manuscript and critically revising it.

Funding Hyderabad Eye Research Foundation.

Competing interests None declared.

Ethics approval LV Prasad Eye Institute.

Provenance and peer review Not commissioned; externally peer reviewed.

(c) Article author(s) (or their employer(s) unless otherwise stated in the text of the article) 2018. All rights reserved. No commercial use is permitted unless otherwise expressly granted.

\section{REFERENCES}

1 Macken PL, Pavlin CJ, Tuli R, et al. Ultrasound biomicroscopic features of spherophakia. Aust N Z J Ophthalmol 1995;23:217-20.

2 Nelson LB, Maumenee IH. Ectopia lentis. Surv Ophthalmol 1982;27:143-60.

3 S D-E. Normal and abnormal development. St Louis: The CV Mosby, 1963.

4 Chan RT, Collin HB. Microspherophakia. Clin Exp Optom 2002;85:294-9.

5 Willi M, Kut L, Cotlier E. Pupillary-block glaucoma in the Marchesani syndrome. Arch Ophthalmol 1973;90:504-8.

6 Johnson VP, Grayson M, Christian JC. Dominant microspherophakia. Arch Ophthalmol 1971:85 534-42.

7 Senthil S, Rao HL, Hoang NT, et al. Glaucoma in microspherophakia: presenting features and treatment outcomes. J Glaucoma 2014;23:262-7.
8 Feiler-Ofry V, Stein R, Godel V. Marchesani's syndrome and chamber angle anomalies. Am J Ophthalmol 1968;65:862-6.

9 Willoughby CE, Wishart PK. Lensectomy in the management of glaucoma in spherophakia. J Cataract Refract Surg 2002;28:1061-4.

10 Muralidhar R, Ankush K, Vijayalakshmi P, et al. Visual outcome and incidence of glaucoma in patients with microspherophakia. Eye 2015:29:350-5.

11 Teekhasaenee C, Ritch R. Combined phacoemulsification and goniosynechialysis for uncontrolled chronic angle-closure glaucoma after acute angle-closure glaucoma. Ophthalmology 1999;106:669-75. (discussion 74-5).

12 Kanamori A, Nakamura M, Matsui N, et al. Goniosynechialysis with lens aspiration and posterior chamber intraocular lens implantation for glaucoma in spherophakia. J Cataract Refract Surg 2004;30:513-6.

13 Harasymowycz P, Wilson R. Surgical treatment of advanced chronic angle closure glaucoma in Weill-Marchesani syndrome. J Pediatr Ophthalmo/ Strabismus 2004:41:295-9.

14 Yang J, Fan Q, Chen J, et al. The efficacy of lens removal plus IOL implantation for the treatment of spherophakia with secondary glaucoma. Br J Ophthalmol 2016;100:1087-92.

15 Chandler PA. Choice of treatment in dislocation of the lens. Arch Ophthalmol 1964:71:765-86.

16 Khokhar S, Pangtey MS, Sony P, et al. Phacoemulsification in a case of microspherophakia. J Cataract Refract Surg 2003;29:845-7.

17 Yasar T. Lensectomy in the management of glaucoma in spherophakia: is it enough? J Cataract Refract Surg 2003;29:1052-3.

18 Senthil S, Rao HL, Babu JG, et al. Outcomes of trabeculectomy in microspherophakia. Indian J Ophthalmol 2014;62:601-5.

19 Kaushik S, Sachdev N, Pandav SS, et al. Bilateral acute angle closure glaucoma as a presentation of isolated microspherophakia in an adult: case report. BMC Ophthalmol 2006;6:29.

20 Taylor JN. Weill-Marchesani syndrome complicated by secondary glaucoma. Case management with surgical lens extraction. Aust N Z J Ophthalmol 1996:24:275-8.

21 Dagi LR, Walton DS. Anterior axial lens subluxation, progressive myopia, and angleclosure glaucoma: recognition and treatment of atypical presentation of ectopia lentis. J Aapos 2006;10:345-50.

22 Singh MS, Casswell EJ, Boukouvala S, et al. PARS plana vitrectomy and lensectomy for ectopia lentis with and without the induction of a posterior vitreous detachment. Retina 2017:1.

23 Hubbard AD, Charteris DG, Cooling RJ, et al. Vitreolensectomy in Marfan's syndrome. Eye 1998;12:412-6. 